\section{Instrument system for monitoring cavitation noise}

\author{
M K De $† \ddagger$ and F G Hammitt \\ $\uparrow$ Department of Nuclear Engineering, The University of \\ Michigan, 550 East University, Ann Arbor, Michigan 48109, \\ USA \\ § Department of Mechanical Engineering and Applied \\ Mechanics, The University of Michigan, 550 East University, \\ Ann Arbor, Michigan 48109, USA
}

Received 31 July 1981, in final form 19 January 1982

\begin{abstract}
The aim of this work is to contribute toward the development of a monitoring system for cavitation damage prediction in hydraulic machinery, particularly high performance centrifugal pumps. A high-frequency pressure-bar probe and digital acquisition and processing system have therefore been designed to measure the characteristics of damaging pressure pulses from cavitation in a venturi which simulates conditions in hydraulic machinery. Measurements were made of pressure pulse height spectra, which were used to develop a cavitation damage prediction method.
\end{abstract}

\section{Introduction}

In the present state of art, it is very difficult or impossible to predict the flow parameters at which cavitation damage will occur in hydraulic machinery. Thus it is highly desirable to have a measurable parameter which will indicate the damaging capability of a given flow regime.

There is a lack of information at present about the complex cavitation phenomenon which is essential for accurate noise diagnosis. Attempts at diagnosing the far-field acoustic noise by measurements of power spectral density alone have prevented previous researchers (Numachi et al 1975, Lush and Hutton 1976, Ramamurthy and Bhaskaran 1979) from identifying some key factors in these processes. The present work is a detailed investigation of the phenomenon. The discrete acoustic pulses from cavitation in a venturi which is part of a high speed water tunnel facility have been investigated by measurements of pressure pulse height spectra. This test facility simulates cavitation in hydraulic machinery. A high frequency pressurebar probe has been designed to measure the amplitudes of the discrete, high frequency, and localised pressure pulses from cavitation. At present there is no commercially available probe for such use. A microprocessor-based data acquisition system was designed and constructed to record the pulse amplitudes measured by the probe, and process them to produce an amplitude distribution function (pulse height spectrum).

The present work, although limited in scope, was conducted to increase understanding of the fundamentals of the above process. The next stage of this work will be directed towards understanding the variation of the observations in various geometries, liquids and flow conditions. These results may then be used for developing a monitoring system for hydraulic machinery.

\$ Present address: Westinghouse Electric Corporation, Water Reactor Divisions, Nuclear Technology Division, PO Box 355, Pittsburgh, Pennsylvania 15230 , USA.
A detailed description of the instrumentation developed and highlights of the results are presented here. A full physical analysis of the results are presented elsewhere (De 1980, De and Hammitt 1982)

\section{Pressure transducer design and observations}

A Kistler 601A commercial transducer and a pressure-bar transducer developed by the authors were used to record the amplitudes of the pressure waves. The characteristics of the two transducers are listed in table 1. The size and frequency response of the commercial Kistler transducer make it less suitable for recording the acoustic waves from cavitation because the typical maximum size reached by bubbles in this venturi is $\sim 1.27 \mathrm{~mm}$ (Ivany 1965), and rise times of the emitted acoustic waves lie in the $1-10 \mu$ s range. Also, the diameter of the liquid jets emitted due to asymmetric bubble collapse is in the range $1 / 10$ th to $1 / 100$ th the maximum bubble radius (Kling 1969).

Figure 1 shows the pressure-bar transducer designed by the authors. The probe was designed specifically for use in this system. Criteria for the design were minimum overshoot, rise time and sensitive head area. Various researchers (Edwards 1958, Jones 1966, Ragland and Cullen 1967) have designed transducers based on the pressure-bar principle for use in shock tubes. Jones and Edwards (1960) studied its response to an underwater spark bubble. This is the first time a probe based on the pressure-bar principle has been used to study natural cavitation in a flowing system.

As shown in figure 1 , the basic component is a cylindrical copper pressure-bar which transmits an acoustic wave without longitudinal reflections until the original wave is reflected from the far end of the bar. This is true because the acoustic impedances of the copper rod $\left(3.2 \times 10^{4} \mathrm{~g} \mathrm{~mm}^{-2} \mathrm{~s}^{-1}\right)$ and the piezoelectric ceramic $\left(3.36 \times 10^{4} \mathrm{~g} \mathrm{~mm}^{-2} \mathrm{~s}^{-1}\right)$ are very close. For a one-dimensional case, the rise time of a step pressure wave is the transit time of the wavefront through the ceramic, in this

Table 1. Characteristics of pressure-bar and Kistler 601A transducers.

\begin{tabular}{lll}
\hline & Pressure-bar & Kistler 601A \\
\hline Sensitive head diameter & $0.0625^{\prime \prime}$ & $0.2^{\prime \prime}$ \\
Rise time & $1.0 \mu \mathrm{s}$ & $3.0 \mu \mathrm{s}$ \\
Maximum temperature & $116^{\circ} \mathrm{C}$ & $260^{\circ} \mathrm{C}$ \\
Sensitivity & $1.65 \mathrm{PCB} / \mathrm{PSI}$ & $1 \mathrm{PCB} / \mathrm{PSI}$ \\
Resonant frequency & $1 \mathrm{MHz}$ & $130 \mathrm{kHz}$ \\
Piezoelectric constant & $374 \mathrm{PCB} / \mathrm{N}$ & $2.3 \mathrm{PCB} / \mathrm{N}$ \\
Mechanical Q & 75 & $10^{6}$ \\
Capacitance (casing) & $7 \mathrm{pF}$ & \\
(ceramic) & $64 \mathrm{pF}$ & \\
(quartz crystal) & & $5 \mathrm{pF}$ \\
\hline
\end{tabular}

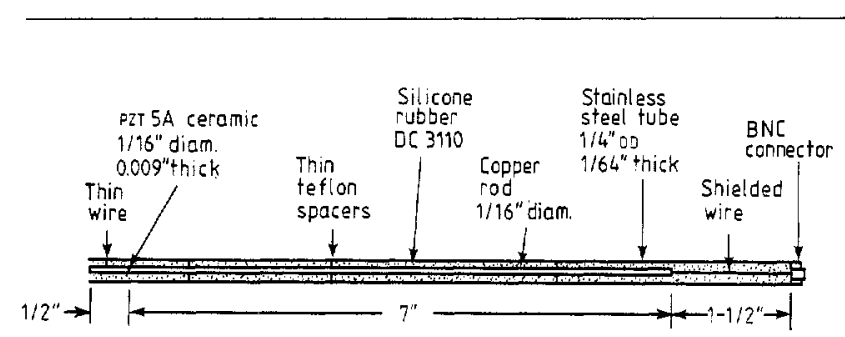

Figure 1. Pressure-bar transducer. 
case approximately $0.05 \mu \mathrm{s}$. In reality, three-dimensional effects will limit the rise time of the observed waveform and cause damped oscillations after the initial response. It has been shown theoretically (Baganoff 1964) that the rise time is proportional to $(v)^{3 / 2}(x / D)^{1 / 3}(D / C)$, where $\nu$ is rod Poisson's ratio, $x$ the distance of the ceramic from the front end of the rod, $D$ the diameter and $C$ the speed of sound in the rod.

If the ceramic is placed at the front end of the bar, then the rise time would be limited by the transit time of the wavefront through the ceramic. Large amplitude oscillations occur in this case due to the reflection of stress waves from the radial boundary of the bar. By constructing and testing probes with different dimensions, a value of 8 for $x / D$ was found to be optimum in the present study. The diameter of the copper rod $\left(1 / 16^{\prime \prime}\right)$ was limited due to fabrication and fragility considerations.

A thin wafer of PZT 5A ceramic was soldered to the copper bars with indium solder ( $50 \%$ indium-50\% tin) which has a low melting point of $116^{\circ} \mathrm{C}$. A low temperature solder was used to avoid damaging the ceramic. The wafer was then ground to shape. The $7^{\prime \prime}(178 \mathrm{~mm})$ backing bar resulted in a reflected wave arriving about $100 \mu \mathrm{s}$ after the original wave. This allows the observation of reflected waves in the venturi, and waves from bubble surface oscillations, as discussed later. Silicone rubber was used to provide mechanical and electrical insulation.

Optimum parameters were selected after testing the transducer with a shock wave emitted by an underwater spark bubble. Condensers, charged from a high voltage power supply, are triggered to discharge into water between two electrodes, in a static spark chamber. Shock waves are emitted by the expansion and implosion of the plasma. The transducers were placed flush with the wall of the chamber. Figures 2 and 3 are oscillographs illustrating the responses of the Kistler 601A and the pressure-bar probes in the spark chamber. The Kistler rises in $2-3 \mu \mathrm{s}$ and then oscillates considerably $(75 \%)$ whereas the pressure-bar probe rises in about $1 \mu$ s with minimal overshoot oscillation $(<25 \%)$. The actual rise time of the shock wave is evidently $<1 \mu \mathrm{s}$ as confirmed elsewhere (Lauterborn and Ebeling 1976).

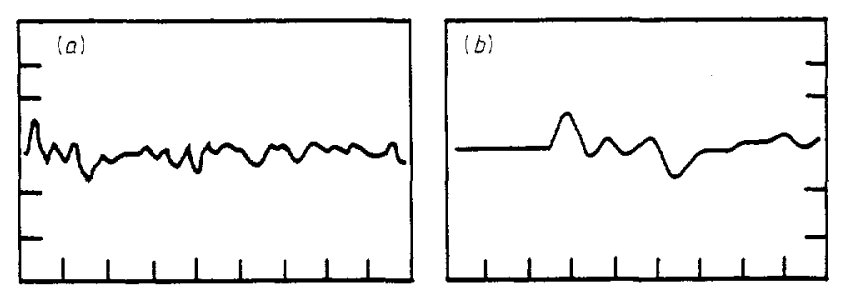

Figure 2. Responses of Kistler (601A) probe to underwater spark bubble: $(a), 1 \mathrm{~V} / \mathrm{div}, 20 \mu \mathrm{s} / \mathrm{div} ;(b), 1 \mathrm{~V} / \mathrm{div}, 10 \mu \mathrm{s} / \mathrm{div}$.

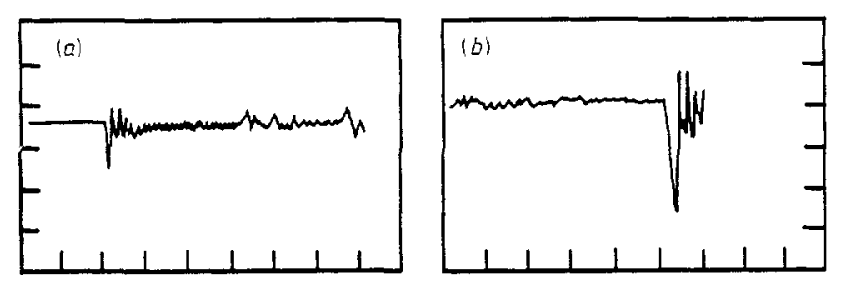

Figure 3. Response of pressure-bar probe to underwater spark bubble: $(a), 10 \mu \mathrm{s} / \mathrm{div}, 0.5 \mathrm{~V} / \mathrm{div} ;(b), 5 \mu \mathrm{s} / \mathrm{div}, 0.2 \mathrm{~V} / \mathrm{div}$.
Figure 4 shows the Plexiglass venturi which is part of a high speed water tunnel facility. Damage specimens and pressure probes can be mounted flush to the wall of the venturi in the same axial plane. The venturi throat velocity and the axial extent of the cavitation cloud is controlled by pump speed and downstream pressure. Figures 5 and 6 show responses of the two probes to bubble collapses and/or rebounds in the venturi. Rise time of the acoustic waves which depends on their origin and intensity is shown to range from $\sim 2$ to $\sim 10 \mu$ s (figures 5 and 6$)$. The slower rise time $(10 \mu \mathrm{s})$ in figure $5(a)$ may be due to a liquid jet impact as observed by Kling (1969) in a similar venturi, rather than shock waves. The Kistler probe does not respond accurately to waves with rise times $<3 \mu \mathrm{s}$ as shown in figure $5(b)$ due to its low resonant frequency. The second pulse in figure $5(b)$ arriving $\sim 80 \mu$ s after the first may be due to a rebound of the bubble. Further evidence, and discussion of these phenomena, are provided later. Figure 6 again shows the minimal overshoot of the pressure-bar probe response. Reflected pulses in the pressure-bar at $\sim 100 \mu$ s intervals are shown in figure 6(a).

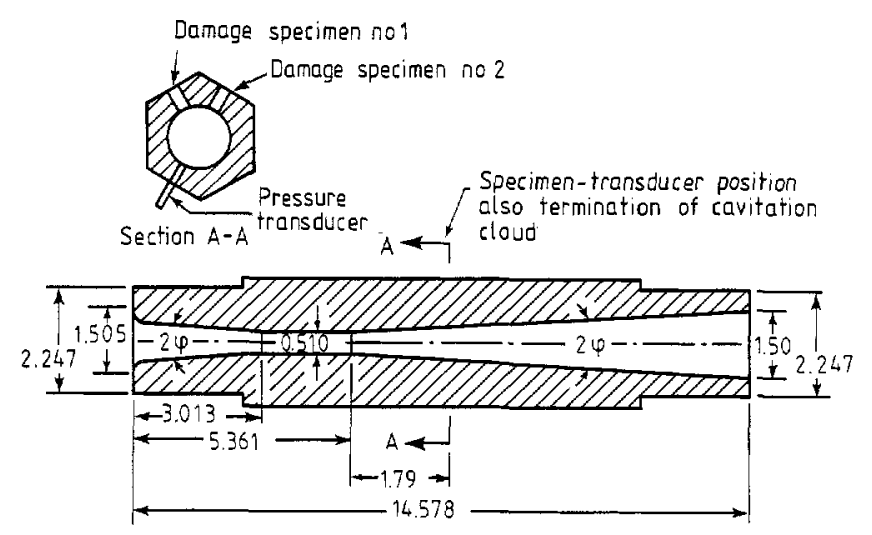

Figure 4. Venturi flow path. $2 \varphi=5^{\circ} 54^{\prime} / 6^{\circ} 04^{\prime}$. All dimensions are in inches.

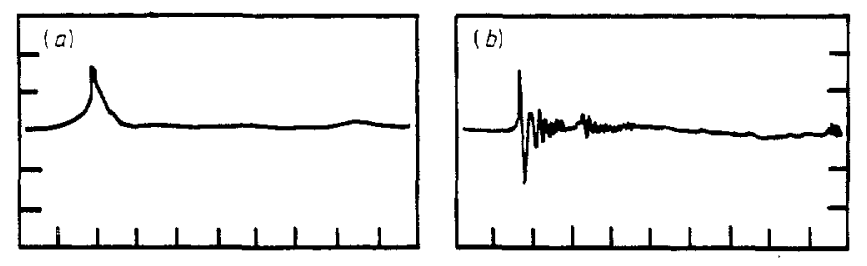

Figure 5. Responses of Kistler (601A) probe to cavitation bubbles in venturi: (a), $50 \mu \mathrm{s} / \mathrm{div}, 0.5 \mathrm{~V} / \mathrm{div}$; (b), $50 \mu \mathrm{s} / \mathrm{div}$, $1.0 \mathrm{~V}$ div.
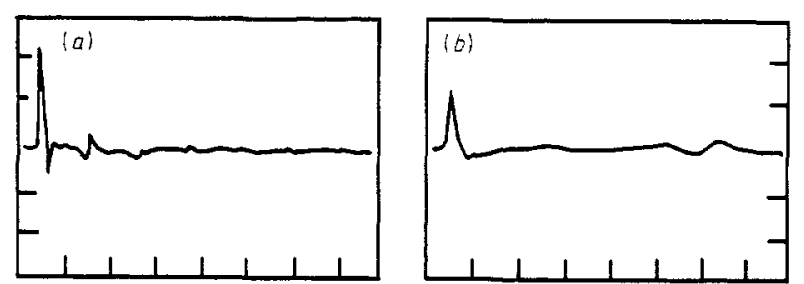

Figure 6. Responses of pressure-bar probe to cavitation bubbles in venturi: $(a), 100 \mu \mathrm{s} / \mathrm{div}, 5 \mathrm{~V} / \mathrm{div} ;(b), 20 \mu \mathrm{s} / \mathrm{div}, 2 \mathrm{~V} / \mathrm{div}$. 
Reflections from the opposite venturi wall appear to be absent. Reflected waves would arrive $\sim 30 \mu$ s after the initial wave and would be attenuated in the water by a factor of $R_{0} / R$, where $R_{0}$ is the maximum bubble radius $(\sim 1.27 \mathrm{~mm})$ (Ivany 1965. De 1980) and $R \cong 20 \mathrm{~mm}$. The reflection coefficient of water to Plexiglass is $\sim 0.2$. The amplitude of the reflected wave would then be $\sim 1 \%$ of the original wave amplitude.

Lauterborn and Ebeling (1976), using Schlieren photography, found that the rise time of such pressure waves is $\sim 0.01 \mu \mathrm{s}$, corresponding to a cavitation shock wave thickness of $\sim 15 \mu \mathrm{m}$ in water. No existing pressure-bar probe, including the one designed here, can determine the duration of such steepfronted shock waves. The probe designed here does, however, measure the approximately correct amplitude as the following discussion will show.

The sensitivity (PSI V ${ }^{-1}$ ) of the pressure-bar probe was calculated in the following manner. The transmission coefficient from water to copper is $\sim 0.08$. The spherical wave will be attenuated due to divergence (Ivany 1965) by a factor of $\sim 10$ in the $0.5^{\prime \prime}(10 \mathrm{~mm})$ front-end bar. By using the combined capacitance of the ceramic, housing, cable and scope, and the piezoelectric constant and surface area of the ceramic, the pressure can be calculated using Coulomb's law, $Q=C V$. The details of the calculation can be found in De (1980).

In the venturi the Kistler 601A (according to manufacturer's calibration) measured peak pressures up to $500 \mathrm{PSI}(3.45 \mathrm{MPa})$. Using the calculational method outlined above, the pressure-bar probe measured peak pressures up to $1.7 \mathrm{GPa}$ in the same venturi. The difference is due to the different sensitive head areas (table 1, factor of $\sim 10$ ) and frequency responses of the probes. The surface area impacted by a typical bubble collapse liquid jet or shock wave would be $\sim 10^{-1}$ to $\sim 10^{-2}$ of the maximum bubble radius (Kling 1969). A typical maximum bubble radius observed (Ivany 1965) in a venturi similar to the one used for the present study is $1.27 \mathrm{~mm}$. Therefore, the surface diameter impacted would be 0.127 to $0.0127 \mathrm{~mm}$. The ratio of the Kistler probe sensitive head diameter (table $1,5.08 \mathrm{~mm}$ ) to a typical impacted diameter is in the range $40-400$. This explains the relatively low pressures indicated by the Kistler (3.45 MPa), whereas pressures needed to cause cavitation damage are no doubt much higher (range $10-10^{2} \mathrm{GPa}$ ) (De 1980) as indicated by the pressure-bar probe. This is the first observation of the peak pressures of waves emitted by collapsing bubbles in a flowing system. Fujikawa and Akamatsu (1978) and others (Jones and Edwards 1960) observed, also with a pressure-bar, that the peak pressure emitted by such bubbles in a stationary system (cavitation bubbles created and collapsed by a shock wave) was in the range $10-10^{2} \mathrm{GPa}$. A calibration of the present pressure-bar probe would be desirable. However, no suitable equipment was available, so that this could not be done as part of the present study. However, the results are in the range found by Fujikawa and Akamatsu (1978) for a similar probe, which was calibrated in a shock tube.

\section{Data acquisition system}

Figure 7 is a block diagram of the digital system used to record and process the amplitude characteristics of the acoustic waves sensed by the probes. The signal from the Kistler transducer is initially amplified in a Kistler 556 charge-amplifier to give peak voltages to $\sim 5 \mathrm{~V}$ in the venturi. The pressure-bar probe senses somewhat higher peaks (to $\sim 10 \mathrm{~V}$ ) due to the higher sensitivity of its ceramic (PZT 5A against quartz), its lack of diaphragm, and its more accurate transient response. The signal may be shunted with a capacitor to reduce temperature effects on the capacitance of the probe.

The cavitation pulses are in general much larger than the background flow noise, which has frequency components up to $\sim 10 \mathrm{kHz}$. An AP-220-5 multifunction filter in high-pass mode

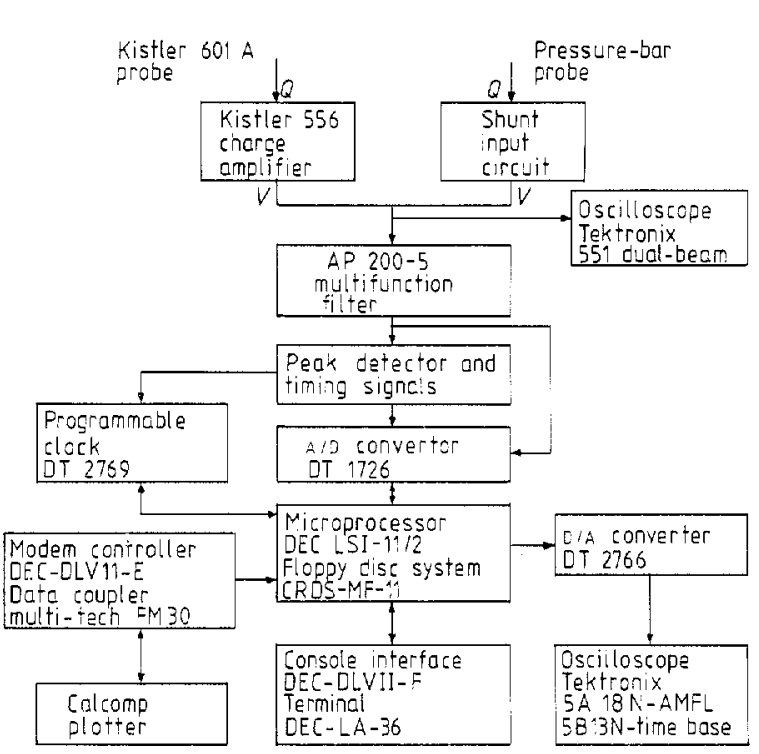

Figure 7. Components block diagram.

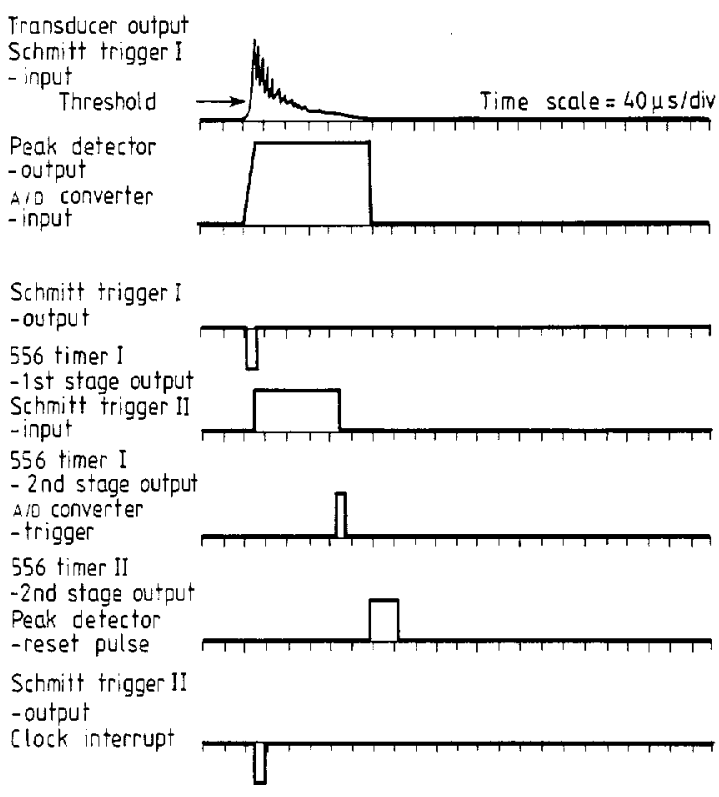

Figure 8. Pressure pulses, peak detector and timing signals.

is used to attenuate the flow noise, when flow system parameters result in reduced cavitation noise. The pulse amplitude is detected by a peak detector designed here with slewrate

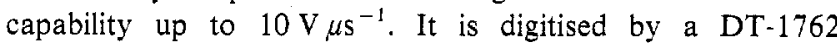
analogue-to-digital converter with timing logic signals generated by a DT-2769 programmable clock, and timing circuitry designed here. Figure 8 shows the sequence and operation of the pressure pulse, peak detector and timing signals.

A potentiometer setting in the programmable clock causes its Schmitt trigger I to fire if the input signal is greater than the set threshold (figure 8). A 556 timer-integrated-circuit is used to produce a delayed trigger pulse to initiate conversion of the peak detector signal to a digital number, which is then sent directly to memory. The time taken for conversion and transfer to memory is $10 \mu \mathrm{s}$. A second 556 timer is used to produce a peak detector 
reset pulse. A dead time is created in which further firings of Schmitt trigger I due to oscillations in the probe output will not result in additional $\mathrm{A} / \mathrm{D}$ trigger pulses.

The original signal may also be digitised directly by periodic trigger pulses out of Schmitt trigger II of the programmable clock. The pulse amplitudes and time intervals are processed with a DEC-LSI-11/2 microprocessor with suitable software.

\section{Typical processed data}

Figures 5 and 6 show that pressure pulses from venturi cavitation bubbles occur discretely in time. To verify this observation an $\mathrm{A} / \mathrm{D}$ converter was used to digitise the original waveform from the Kistler $601 \mathrm{~A}$ transducer to study the pressure pulses over a period of $250 \mathrm{~ms}$. Since the sampling rate was limited to $70 \mathrm{kHz}$, a low-pass filter set at $50 \mathrm{kHz}$ was used to attenuate the $130 \mathrm{kHz}$ resonant frequency of the transducer, also resulting in the attenuation of the peak pressures of the cavitation pulses by $24 \mathrm{~dB} /$ octave from the cut-off point $(50 \mathrm{kHz})$. Figure 9 shows a typical segment of the data, running from 0 to $4.2 \mathrm{~ms}$. It should be noted that the pulse amplitudes are in reality much larger than the background flow noise, and are indeed discrete. The repeated signal at about $10 \mathrm{kHz}$ (figure 9) confirms that some of the bubbles do indeed oscillate, as indicated earlier. This phenomenon has been photographically observed here by Ivany and Hammitt (Ivany 1965) in a similar venturi. The oscillation frequencies $(5.0$ to $20.0 \mathrm{kHz}$, figure 9) correspond to the natural frequencies of bubbles observed by Ivany and Hammitt, which ranged in diameter from 0.254 to $1.27 \mathrm{~mm}$. The oscillations may be excited by turbulent pressure fluctuation in the liquid. The pulse from bubble rebound (Ivany 1965, Hickling and Plesset 1964) is sometimes larger than that from bubble collapse as shown in figure 9 .

Further work is planned to obtain statistics of the occurrences of collapses, rebounds, oscillations, liquid jet impacts, and the spatial origin of the bubbles. This can be achieved by modifying the hardware and software for a dualchannel system. Outputs of two probes $180^{\circ}$ apart, in the same axial plane, can then be simultaneously recorded.

Figure 10 shows a typical pulse height spectrum measured by the Kistler transducer. The vertical lines (figure 10) indicate

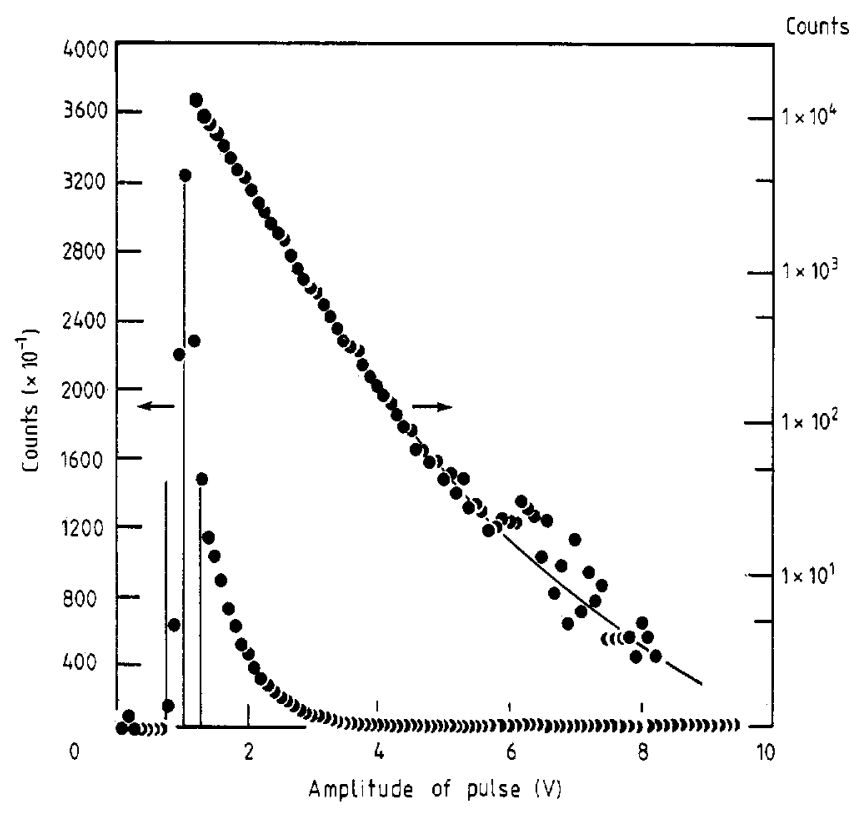

Figure 10. Typical pulse height spectrum. $(1 \mathrm{~V}=0.36 \mathrm{MPa}$.

the variation in the threshold setting shown in figure 8 . In figure 10 an exponential decay of the spectrum is shown followed by a power law variation. The decay constant of the spectrum acquired with the Kistler was found to be about a factor of two smaller than that acquired with the pressure-bar probe at the same flow conditions. Some difference would be expected since the Kistler probe will record less correctly a large pressure pulse, if the bubble collapses close to its relatively large diaphragm. The decay 'constant represents information about the probability distribution of the pulses in the spectrum, but does not indicate anything about their amplitudes. The Kistler probe functions fairly well in acquiring the relevant shape of the spectra, but fails to record the correct absolute amplitudes of the pressure waves.

Pulse amplitude (V)
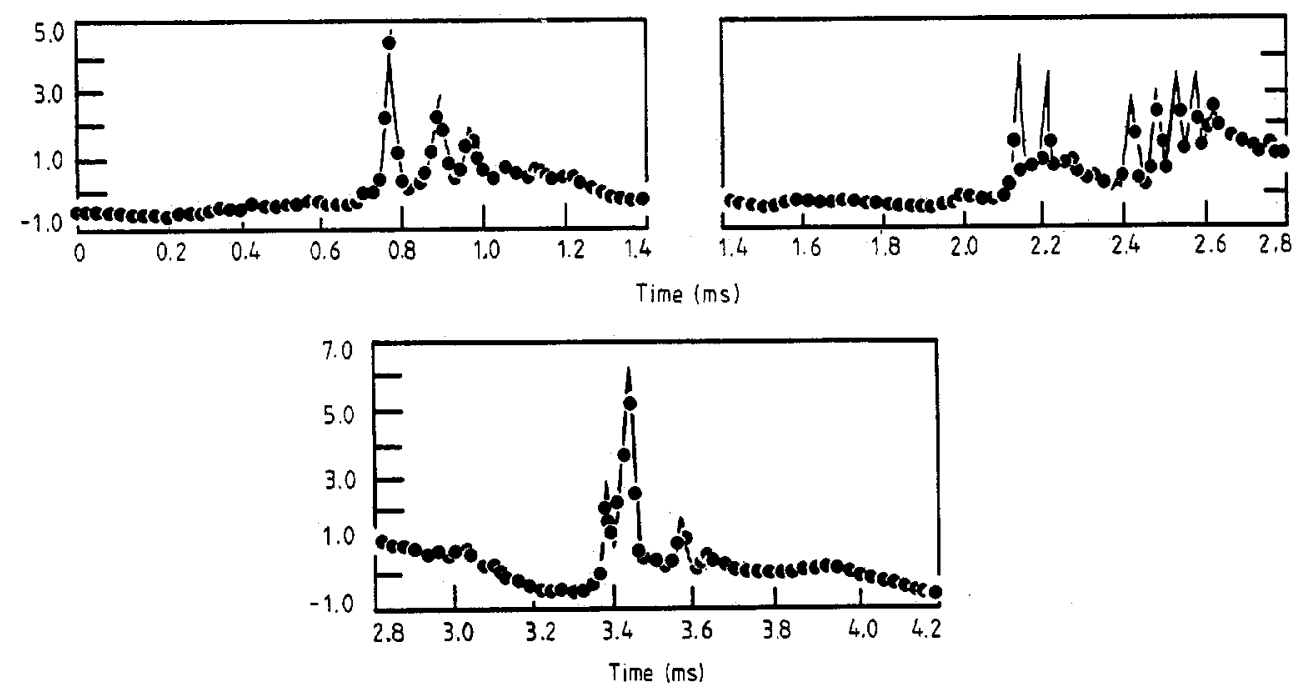

Figure 9. Typical digitised cavitation pressure pulses. 
It has been found that the area under a pulse height spectrum curve can be correlated to cavitation damage and frequency shifts in the power spectrum, thus possibly allowing damage predictions in pumps from a priori correlations.

Complete results obtained by this instrumentation system, theoretical formulations, and explanations of the observed results can be found elsewhere (De 1980, De and Hammitt 1982).

\section{Conclusion}

A pressure-bar probe and a digital data acquisition and processing system have been developed, and successfully used to find the amplitude characteristics of pressure pulses from flow cavitation. The observations presented here in detail show that cavitation bubbles incept discretely, and some of the bubbles oscillate at frequencies in the range 5 to $20 \mathrm{kHz}$. These results, and other observations briefly presented here, provide knowledge about cavitation inception, growth and collapse that will be useful for developing a monitoring system to predict cavitation damage in hydraulic machinery.

\section{Acknowledgments}

We wish to thank Mr W Kittle for his advice on the design of the data acquisition system and Messrs F Smith and $\mathrm{H}$ Senecal for their help in constructing the pressure-bar probe.

Financial support was provided by Office of Naval Research Contract N00014-76-C-0697, NSF Equipment Grant No. ENG-78-07997, internal University of Michigan SEP and Work Study funds.

\section{References}

Baganoff D 1964 Pressure gauge with one-tenth microsecond risetime for shock reflection studies

Rev. Sci. Instrum. 35288

De M K 1980 Acoustic waves from hydrodynamic cavitation $P h D$ Thesis University of Michigan

De M K and Hammitt F G 1982 New method for monitoring and correlating cavitation noise to erosion capability

Trans. ASME, J. Fluids Engr. to be published

Edwards D H 1958 A piezo-electric pressure-bar gauge

J. Sci. Instrum. 35 346-9

Fujikawa S and Akamatsu T 1978 Experimental investigations of cavitation bubble collapse by a water shock tube Bull. JASME 21 No $152223-30$

Hickling R and Plesset M S 1964 Collapse and rebound of a spherical bubble in water

Phys. Fluids 7 7-14

Ivany R D 1965 Collapse of a cavitation bubble in viscous, compressible liquid-numerical and experimental analyses PhD Thesis University Michigan

Jones I R 1966 Beryllium pressure bar having submicrosecond risetime

Rev. Sci. Instrum. 37 1059-61

Jones I R and Edwards D H 1960 An experimental study of the forces generated by the collapse of transient cavities in water J. Fluid Mech. 7596

Kling C L 1969 A high-speed photographic study of cavitation bubble collapse

\section{PhD Thesis University of Michigan}

Lauterborn W and Ebeling K J 1976 High speed holography of laser-induced cavitation bubbles in liquids Proc. 7 th Int. Symp. Non-linear Acoustic, August 19-21, Blacksburg, VA USA (New York: Acoustical Society of America)

Lush P A and Hutton S P 1976 The relation between cavitation intensity and noise in a venturi-type section Proc. Int. Conf. on
Pump and Turbine Design, Glasgow, Scotland, September 1976 Numachi F, Chida I and Hongo M 1975 Ultrasonic shock wave emitted by cavitation at perforation on plate Rep. Inst. High Speed Mech. 32 No 283

Ragland K W and Cullen R E 1967 Piezoelectric pressure transducer with acoustic absorbing rod

Rev. Sci. Instrum. 38 740-2

Ramamurthy A S and Bhaskaran P 1979 Velocity exponent for erosion and noise due to cavitation Proc. ASME/CSME Appl. Mech., Fluids Engr. and Bioengr. Conf., Niagra Falls, NY, June 1979 (New York: American Society of Mechanical Engineers) 